\title{
THE EFFECT OF CARDIAZOL CONVULSIONS ON THE DISTRIBUTION AND ACTIVITY OF SOME PHOSPHATASES IN THE AREA POSTREMA OF THE RAT
}

\author{
BY \\ JORGE A. INSUA* and D. NAIDOO \\ From the Department of Neuropathology, Maudsley Hospital, University of London
}

During previous studies on the localization of phosphatases in the central nervous system of the rat it was observed that the area postrema showed intense enzyme activity to a number of important phosphoric acid esters in the acid and alkaline ranges. This structure therefore provided a convenient point upon which to test the effects in situ on some brain enzymes of substances used in neuropsychiatric therapy.

Since anatomical information on the area postrema of the rat was limited, a preparatory study of the medulla was undertaken to establish the normal histology. Cardiazol was the test substance of choice because accurate doses could be administered intravenously and because the subsequent convulsions could be assessed readily according to length and severity.

\section{Material and Methods}

Preparatory anatomical and histological work was carried out on 22 adult albino rats weighing between 200 and $250 \mathrm{~g}$. The methods applied were the ordinary histological staining procedures including various silver impregnation techniques.

A histochemical study of the area postrema was made on the frozen-dried brains of 45 adult albino rats. The femoral veins of anaesthetized rats were exposed and $0.05 \mathrm{~g}$. of "cardiazol" in a $10 \%$ solution in double-distilled water was injected. The convulsions began immediately and the animal was transferred to a chamber into which a small constant flow of oxygen was directed to avoid anoxia. The rats were in convulsions between a half and four hours. Control animals were injected with an equal volume of distilled water and placed in the same atmosphere. An experimental animal and its control were anaesthetized together and both brains were removed rapidly. The cerebellum was then cut through approximately between its anterior two-thirds and posterior third. The caudal block containing the area postrema was immediately dropped into isopentane cooled to below $-65^{\circ} \mathrm{C}$. in a bath of acetone and $\mathrm{CO}_{2}$ ice.

\footnotetext{
* British Council scholar.
}

After about two to five minutes the materia! was transferred to a drying tube made of pyrex glass $15 \mathrm{~cm} . \times$ $3 \mathrm{~cm}$. provided with a B34 ground socket. The tube was previously filled to the level of about $2.5 \mathrm{~cm}$. with paraffin wax (m.p. $52^{\circ} \mathrm{C}$.) and cooled to $-78^{\circ} \mathrm{C}$. in the acetone and $\mathrm{CO}_{2}$ ice bath. The material was placed on this wax bed. The tube was then affixed to the vacuum pump (2.S.50 Edwards) by means of a glass wide-bore adaptor. Between adaptor and pump was interposed anv isolation valve and a $\mathrm{P}_{2} \mathrm{O}_{5}$ trap. The tube was then surrounded by a bath (a 2-litre Dewar flask) of phenyl ethyl-ether cooled to about $-75^{\circ} \mathrm{C}$. with $\mathrm{CO}_{2}$ ice. The pump was started and the process of dehydration began. The phenyl-ethyl-ether bath was allowed to rise to about $-30^{\circ} \mathrm{C}$. at which temperature it was maintainedo by the addition of $\mathrm{CO}_{2}$ ice. The pressure was frequentlyo measured by a hot-wire Pirani gauge incorporated with the adaptor connecting tissue tube to the vacuum pump The working pressure was about 0.02 to $0.002 \mathrm{~mm}$. $\mathrm{Hg}$ and the time required for adequate drying was about 72 hours. When this period of time had elapsed the temperature was allowed to rise to room temperature by removal of the refrigerant. After 30 minutes at room temperature the pressure in the tube containing tissue was brought up to between 2 and $10 \mathrm{~mm}$. $\mathrm{Hg}$ by the introduction of dry air. Having done this, the paraffin bed in the tube was slowly melted by surrounding the tube with a warm water bath. In this manner vacuum embedding in dry air was obtained. The temperature of the wax was not allowed to rise beyond about $56^{\circ} \mathrm{C}$. After wax penetration for 20 minutes the tube was removed and the blocks embedded in paraffin of the same melting point as that which made up the wax bed. These blocks were stored at $0^{\circ} \mathrm{C}$. in a dessicator. The tissue blocks were cut within one month of embedding. Sections $15 \mu$ thick were mounted on albuminized $3 \times$ 1 in. slides without floating on water. The sections were placed in a $37^{\circ} \mathrm{C}$. incubator for 60 minutes. De-waxing was carried out by successive immersions for less than two minutes altogether in light petroleum (b.p. 100 to $120^{\circ}$ ), petroleum (b.p. 60 to $80^{\circ}$ ), and isopentane (b.p. 27 to $31^{\circ}$ C.). The last solvent evaporated immediately after the section was removed from the de-waxing jar. The sections were placed at once in the prepared sub- 
strate media contained in coplin jars. The media were made up as the following formulae indicate.



The preparation of adenosine triphosphate, adenosine monophosphate, and of the buffer solutions followed the methods advised by Naidoo and Pratt (1951 and 1952a or b).

The substrate medium was degassed immediately before the introduction of the sections. In order to ensure further that no gas bubbles would adhere to the surface of the tissue the slides were flicked into the medium with a sharp movement of the fingers so that they went in very rapidly. Incubation proceeded for intervals of $3,10,20,40,80$, and 120 minutes. The sections were then removed, washed in four changes of ice-cold, glass-distilled water, and then placed for two minutes in $2 \%\left(\mathrm{NH}_{4}\right)_{2} \mathrm{~S}$ solution.

A comparison between the experimental and control series was made by cutting ribbons of sections on a rotatory microtome from single blocks of each series, and one anatomically similar section each from two comparable ribbons from separate blocks were incubated together on the same slide at the same level on the slide. Three separate criteria were employed in assessing histological enzymatic activity. First, a histological comparison of both sections incubated for the same time, secondly the difference in incubation time required to produce an equal depth of staining (Naidoo and Pratt, 1953), and thirdly a comparison of the response to inhibitors used with the normal control and the experimental material (Naidoo and Pratt, 1953).

\section{Results}

Anatomical and Histological.-The ependymal conus (area postrema) of the rat starts caudally at a point some $650 \mu$ below its cephalic margin at the midpoint of the dorsal median raphe behind the commisural nucleus of Cajal. Vessels from the posterior median fissure are directed towards this site which is made up of mesodermal and glial fibres. About $150 \mu$ rostral to this point the conus is larger, round, and its ventral limits are clearly demarcated by a dense glial wall whereas laterally the borders are indistinct. Further forward the absence of a definite lateral boundary gives the impression that the structure of the conus becomes intimately associated with that of the nucleus solitarius. At this point the central canal becomes T-shaped in cross section and the conus more wedge-shaped. There is a thick glial fibre bundle connecting the central part of the ventral surface of the conus to the central canal. In between these fibres there are some astrocytes and ependymal cells. As the restiform bodies diverge and the fasciculus solitarius with them, the conus is stretched out into a fan-shaped structure whose handle lies in the roof of the central canal. Further cephalad the stretched conus forms the roof of the central canal but the anterior boundary is reached soon after the conus becomes an intraventricular structure. At each lateral end of the anterior boundary, which may be visualized as the base of a triangle, a short, thick prolongation of the conus is seen.

The conus, then, is dorsally related to the central canal and connected to its ependymal lining by a ventral glio-ependymal prolongation from it ; it is related, by making contact with it, to the nucleus solitarius by a prolongation running laterally below the posterior funiculi of the medulla. The nucleus solitarius lies between this prolongation and the dorsal nucleus of the vagus. The intermediate zone between the pars magnocellularis of the nucleus solitarius and the ventrolateral border of the ependymal conus is occupied by what has been called the subnucleus tracti solitarii parvo cellularis by Meesen and Olszewski (1949).

The boundaries of the conus are definite except antero-laterally and at the ventral midpoint where prolongations exist. It is a highly vascular and cellular area. The vascular arrangement is that of a sinusoidal bed in which are set, among the sinusoids, astroglia, microglia, fibroblasts, and a type of cell containing a large oval clear nucleus with often an eccentric nucleolus and a small amount of chromatin. The larger of the cells lie at the periphery. Bielschowsky's silver technique reveals a very small number of protoplasmic processes. The astrocytic processes are not traceable readily to the walls of the sinusoids. Large amounts of collagen are seen around the vessels and the glial wall delimiting the conus is seen to be built up by processes of fibrous astroglia. Extensive search after the use of various silver techniques failed to demonstrate any structure 




FIG. 1

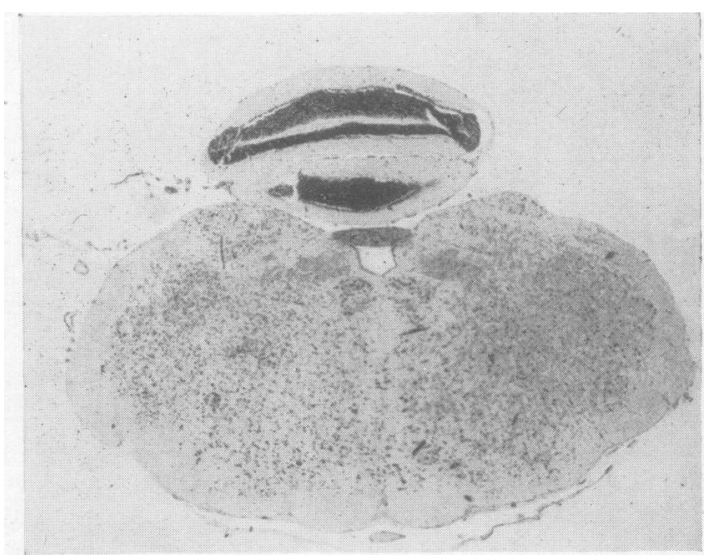

FIG. 2

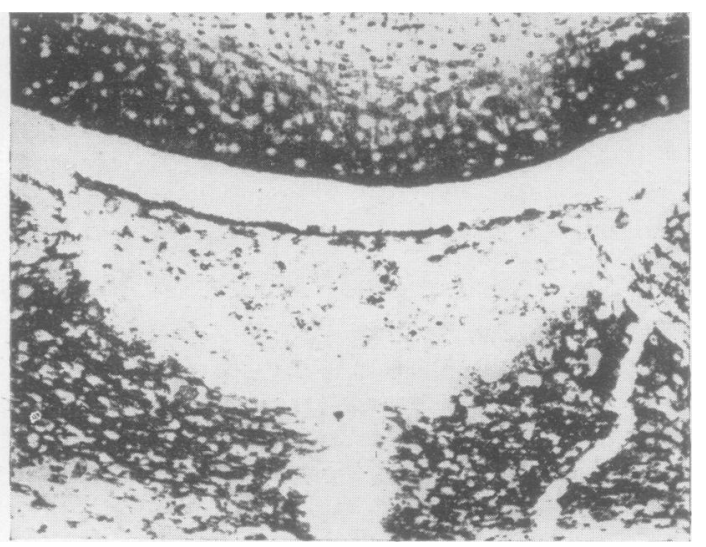

FIG. 3



FIG. 4

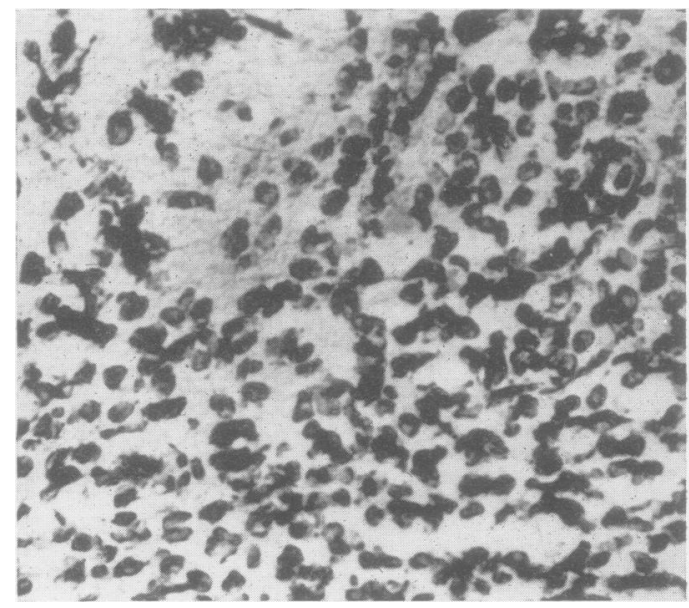

Fig. 5

FIG. 1.-Area postrema showing relation to medullary structures. $\times 26$. Heidenhain stain.

FIG. 2.-Anterior end of area postrema showing bridge over central canal at apex of the fourth ventricle. $\times 14 . \quad$ Nissl stain.

FIG. 3.-Adenosine-monophosphatase $p H$ 6.5: relatively no activity. $\times 90$.

FIG. 4.-Glycerophosphatase $p \mathbf{H}$ 9.1 : intense activity of area postrema. $\times 12$.

FIG. 5.-Aneurinpyrophosphatase $p H$ 6.9 showing strong activity in parenchymal cells. $\times 350$

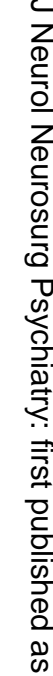

$\overrightarrow{0}$

$\overrightarrow{\vec{\omega}}$

?.

$\nabla \dot{\omega}$

윰요

을

б叉

8 은

象

흘

ํํㅇำ

윽

祍

产

훅

ᄋิ

윽

궁

을.

N

N

స్ట

श्र

$\stackrel{0}{\stackrel{0}{D}}$ 




FIG. 6

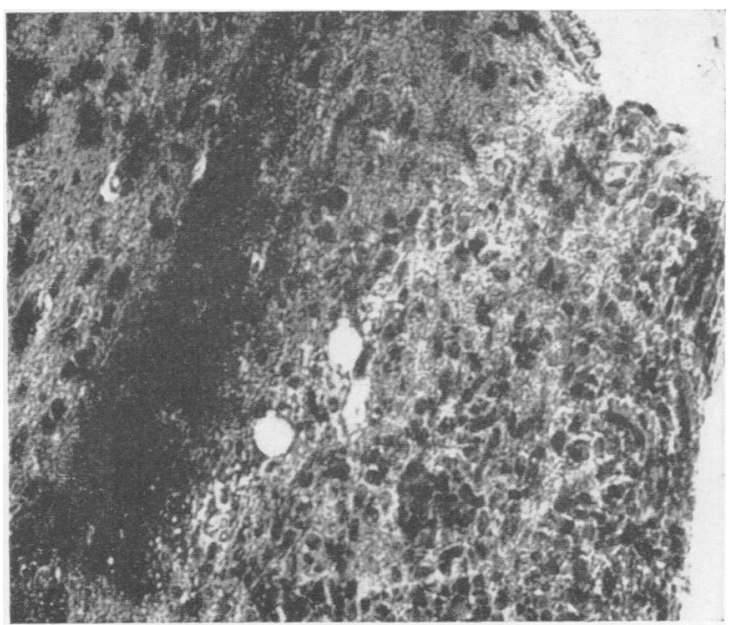

FIG. 8

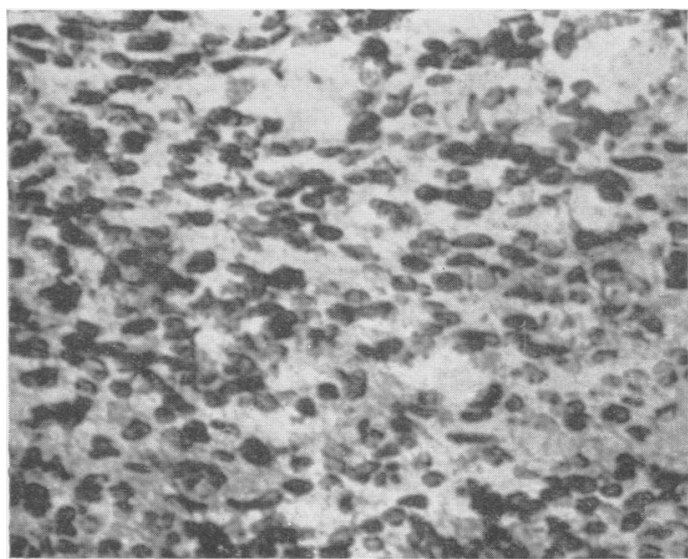

FIG. 9

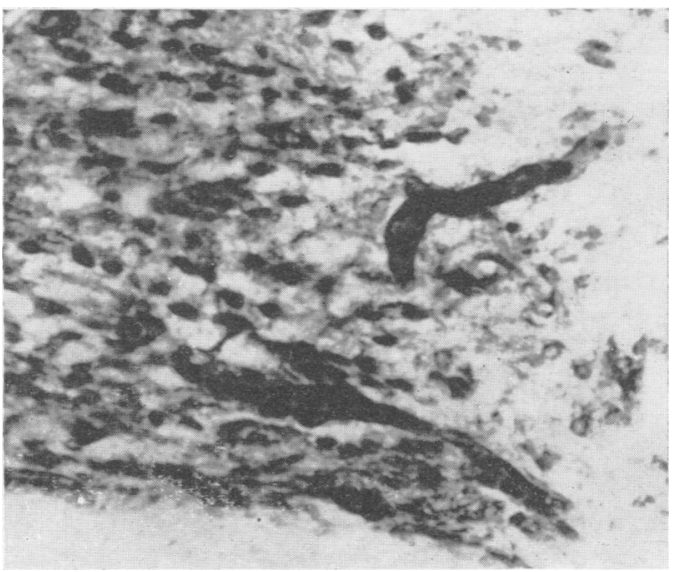

Fig. 10 
which could be indubitably described as an axon. Reticulin methods show abundant reticular fibres around the sinusoids and a thick layer in the outer surface of the conus. The larger cells with oval nuclei, which form the main cell of the parenchyma of the area postrema, appear to be modified cpendymal cells.

Adenosine-monophosphatase : Normal.-The hydrolysis of adenosine monophosphate is found to be situated in the fibres of the medulla but by sharp contrast the area postrema stands out as a clear, unstained triangular area (Fig. 3). The outer border of the conus, however, appears a little more deeply stained than the remaining fibres of the medulla. Within the conus itself it is difficult to discern any staining of separate cellular elements. Here and there a short, thin fibre can be seen to be stained. At the longer incubation periods the cells, particularly those described above as parenchymal cells, begin to show slight staining. Nucleolar staining is inconsistent and appeared only in a few cells. The main impression is the absence of hydrolytic activity to adenosine monophosphate in the area postrema.

Adenosine-monophosphatase after Cardiazol.-The histochemical observations on the area postrema of animals convulsed with "cardiazol" show that the sites of hydrolysis of adenosine monophosphate in the area postrema are unaltered after convulsions. The area remains clearly demarcated as an unstained wedge. The rate of staining in the fibres of the medulla shows no change.

Glycerophosphatase $p H$ 9.1 and 5.3 : Normal.When sections which have been incubated with glycerophosphate at $p \mathrm{H} \mathrm{9 \cdot 1}$ are examined, the conus appears after 40 minutes' incubation as a black triangle set in a grey mottled background of medulla (Fig. 4). The conus thus shows intense enzyme activity with this substrate. The activity is so great that a positive result was obtained after an incubation period of two minutes. At such short incubation periods activity of the capillary endothelium can be distinctly demonstrated. Longer incubation periods increase the staining of the capillaries and the intersinusoidal parenchyma also begins to turn grey. The greyness does not start in the cells or collagen but is general and evenly distributed over the whole area. The area postrema and the choroid plexuses are the parts of the brain which stain most deeply after incubation with

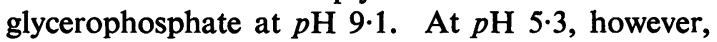
the localization of the sites of enzyme activity differ from those found in the alkaline $p \mathrm{H}$. In the area postrema the parenchymal cells are stained but not the sinusoids or glial cells. The parenchymal cells themselves appear to vary in their reaction to glycerophosphate. Some stain much more deeply than others over the same incubation period. That segment of tissue described as the intermediate zone (subnucleus tracti solitarii parvo-cellularis) between the conus on the one hand and the vagal and hypoglossal nuclei on the other, also shows strong enzyme activity (Fig. 6). The nuclei of the vagus and hypoglossal nerves show neuronal staining and in the longer incubation periods some parenchymal staining as well. The neurons show deep cytoplasmic staining, a clear nucleus, and a stained nucleolus. At the level of the fibroglial boundary there is a clear demarcation between the homogeneously stained intermediate zone and the conus in which only the parenchymal cells are stained.

Glycerophosphatase after Cardiazol.-In the sections incubated in glycerophosphate at $p \mathrm{H} 9 \cdot 1$ the reaction in the tissue from convulsed animals is considerably accelerated. After an incubation period of two minutes the capillaries in the experimental tissue are much darker than those of the control tissue. The whole area also appears darker : no definite cytological elements can be seen in the intercapillary spaces but the parenchyma generally takes part in the increased rate of staining. Even against this darker background the staining of the capillary walls is proportionately greater. After long incubation periods of one hour or more the differences become less discernible and by 90 minutes both normal and experimental areae postremae are equally black. At $p \mathrm{H} 5.3$ the area postrema of the experimental animal stains considerably less than that of the control. In those animals in which convulsions were of relatively short duration-about half-an-hour-before the removal of the brain, the intermediate zone (subnucleus tracti solitarii pars parvocellularis) stains deeply but when the convulsions are prolonged up to about four hours the intermediate zone as well as the conus fails to stain. The longer the animal remains in a state of convulsion the more slowly does the intermediate zone hydrolyse glycerophosphate at $p \mathrm{H} 5 \cdot 3$. In the normal the neurons of the cranial nerve nuclei only showed staining of the cytoplasm of the nucleolus and of some karyosomes, whereas in the experimental animal the processes from the cells were also stained for a short distance from the cell body.

Vitamin $B_{1}$ Diphosphatase $p H \quad 6.9$ and $9 \cdot 1$ : Normal.-When the sections are incubated with aneurin pyrophosphate (vitamin $\mathbf{B}_{1}$ diphosphate) 
they present quite a different picture. The area postrema and both parts of the nucleus solitarius are deeply stained whereas the nuclei of the vagus and the hypoglossus are only lightly stained. In the area itself only a few of the parenchymal cells show activity (Fig. 5). Those parenchymal cells which demonstrated staining show that their cytoplasm as well as their nuclei and karyosomes stained. Between these cells of the conus, the vessels stand out clearly because of the deep staining of the endothelium. The vessels appear at moderate and long incubation periods. The glial cells stain lightly. In the alkaline range the vessels are distinctly stained but in the acid $p \mathrm{H}$ no vessels at all are seen. The cranial nerve nuclei stained are similar to those found in the acid range. The details of neuronal staining are clearly seen in the large neurons of the cranial nerve nuclei. The cytoplasm and the nucleolus are most deeply stained and the nucleoplasm is consistently a little more lightly stained. Scattered throughout the nucleoplasm is a number of discrete granules of deeply staining material. In the cytoplasm the staining appeared to be deepest in some aggregates of amorphous material. It is difficult to say whether these bodies represented Nissl substance.

Vitamin $B_{1}$ Diphosphatase after Cardiazol.-The hydrolysis of vitamin $B_{1}$ pyrophosphate is less than the normal control after "cardiazol" convulsions. The difference in staining is seen most clearly at incubation periods of 20 to 30 minutes (Fig. 9). At $p \mathrm{H}$ $9 \cdot 1$, however, a further difierence is seen in the vessels, which, in tissue from animals injected with "cardiazol", stain much more deeply than the definite but lightly stained vessels of the con'rol animal (Fig. 10).

Adenosinetriphosphatase: Normal.-In the material incubated with adenosinetriphosphate, incubation periods of 20 minutes are needed to show even very light staining. The nuclei of the nerve cells and the glial cells of the medulla appear at this length of incubation (Fig. 7). No vessels or other elements are seen to be stained. In the area postrema the nuclei and nucleoli of cells irrespective of type appear.

Adenosinetriphosphatase after Cardiazol.-No alteration in the rate or sites of adenosinetriphosphatase activity was detected in the area postrema after convulsions. Longer incubation periods increased the staining of the nuclei but no new structures could be seen. The parenchymal cells of the conus showed distinct, large, light- to deep-staining nuclei with deeply stained nucleoli. After a 40-minute incubation the nuclei and nucleoli of the cranial nerve neurons are clearly stained but the cytoplasm of the cells whether neuronal or ependymal do not take part in the hydrolysis of adenosinetriphosphate.

\section{Discussion}

Histochemically the area shows a consistent and definite pattern of enzyme activity. Adenosine monophosphate is generally hydrolysed in the nerve fibres. It has been shown previously that the cell body is free from any hydrolytic properties for this substrate. Nucleolar staining should, at present, be regarded as a possible artefact of the method. The nucleolus may form a focus of crystallization or have a special affinity for the phosphate freed from the phosphoric acid ester. The area postrema appears to have no nerve fibres and consequently no enzyme activity for adenosine monophosphate.

It is possible that the occasional short fibre, rarely more than one or two in an entire section of the area, may be a nerve fibre with its cell body elsewhere. The hydrolysis of glycerophosphate at $p \mathrm{H} 9 \cdot 1$ shows that this alkaline phosphatase is practically confined to the sinusoidal wall. Much has been written about the role of alkaline phosphatase in glycolysis (Richter, 1951) and in the processes of contractility carried out by proteins (Fell and Danielli, 1943). Its bilaminar arrangement in the intestinal mucosa (Johnson and Kugler, 1953) has given weight to hypotheses concerning the phosphorylation and transport of intestinal glucose. The possibility that the sinusoids of the area postrema may be especially sensitive to metabolites in the blood on the one hand, or to changes in the closely applied parenchymal cells on the other, arises. The direction of the processes assisted by enzyme action in the sinusoidal wall could conceivably be bidirectional : from the blood into the parenchyma as well as from the parenchyma into the circulation.

Examination of the cerebral cortex and other areas showed in earlier work that acid phosphatase at $p \mathrm{H} 5.3$ with glycerophosphate as substrate is active strongly in the cytoplasm of neurons. Bodian and Mellors (1944) have shown that the activity of this enzyme increases during the degeneration and regeneration of Nissl substance. The parenchymal cells are not all active in the sections and there is a suggestion that those closest to the sinusoids are most prominent in their staining. As the sinusoidal bed is very dense, this point could not be clarified further. More interesting, however, is the staining of a zone of brain tissue representing the small-celled part of the nucleus solitarius lying between the conus and the vagal and hypoglossal nuclei, called for convenience the intermediate zone. Previous 
studies (Naidoo and Pratt, 1951) show acid phosphatase (glycerophosphatase) to be a cytoplasmic enzyme, but this small zone shows staining of cells and tissue between the cells as well. The staining extends from the border of the conus to the vagal and hypoglossal nuclei. The neurons of the vagal and hypoglossal nuclei themselves show the typical cytoplasmic staining observed in earlier studies. Banga, Ochoa, and Peters (1939) found aneurin to be more effective in passing the permeability barriers than the diphosphate, and it is possible that in this form it enters the cells, takes part in the oxidative decarboxylation of pyruvic acid, so that metabolism passes beyond the pyruvate stage. The dephosphorylation of vitimin $B_{1}$ diphosphate occurs in the area postrema in both the acid and alkaline ranges. At the alkaline $p \mathrm{H}$ the vessels as well as the parenchymal cells take part in the dephosphorylating process. The separate identity of glycerophosphatase has already been established. The enzyme hydrolysing glycerophosphate at $p \mathrm{H} 9 \cdot 1$ was shown to be chemically and histologically distinct from the enzyme hydrolysing aneurin pyrophosphate at the same $p H$ (Naidoo and Pratt, 1953). Adenosine triphosphate is an energy-rich phosphate. The rupture of the pyrophosphate bonds is now believed to yield considerable quantities of energy. It was previously found that in all regions of the brain the nuclei of cells of all types showed activity in this substrate. The findings for the area postrema do not differ.

After "cardiazol" the cleavage of glycerophosphate in the alkaline range is considerably accelerated whereas the hydrolysis of the same substrate in the acid range is retarded. The increase in alkaline phosphatase activity is restricted mainly to the capillary endothelium where activity normally takes place, whereas the function of acid phosphatase is successively reduced in the area postrema and the intermediate zone depending on the length of convulsions. Aneurin pyrophosphate is broken down more slowly after convulsions than by normal tissue in the acid range, but in the alkaline range an additional factor is that the sinusoids stain more prominently than in the control. It is possible therefore that non-specific alkaline phosphatase activated by "cardiazol" convulsions acts upon aneurin pyrophosphate, breaking it down in the capillaries where the enzyme is situated.

The rest of the medulla seen in each preparation of the area postrema was also studied to exclude the possibility that the differences seen were general differences applicable to the medulla as a whole. Changes in the cells of the reticular formation were not sought. The changes described were limited to the area postrema except for the increased activity of alkaline phosphatase which appeared in the sinusoids of the area postrema as well as in the capillaries of the medulla.

\section{Summary}

The effects of " cardiazol " convulsions upon the distribution and activity of enzymes hydrolysing adenosine triphosphate, adenosine monophosphate, aneurin pyrophosphate, and glycerophosphate in the area postrema of the rat are described.

There are clear differences between the cytological enzyme activity observed in the normal animal and in the animal injected with "cardiazol".

The significance of the normal distribution of enzyme activity and of the changes after " cardiazol " convulsions is discussed.

We wish to thank Professor A. Meyer for his support of and interest in this work, and the Bethlem Royal and Maudsley Hospital Research Fund for financial support. We wish to thank also Dr. O. E. Pratt for his collaboration in the purification of some of the substrate materials used.

\section{REFERENCES}

Banga, I., Ochoa, S., and Peters, R. A. (1939). Biochem. J., 33, 1109. Bodian, D., and Mellors, R. C. (1944). Proc. Soc. exp. Biol., N.Y., $55,243$.

Fell, H. B., and Danielli, J. F. (1943). Brit. J. Exp. Path., 24, 196.

Johnson, F. R., and Kugler, J. H. (1953). J. Anat., 87, 247.

Meesen, H., and Olszewski, J. (1949). A Cytoarchitectonic Atlas of the Rhombencephalon of the Rabbit. Karger, Basel.

Naidoo, D., and Pratt, O. E. (1951). Journal of Neurology, Neurosurgery and Psychiatry, 14, 287.

(1952a). Ibid., 15, 164.

(1952b). First Congress of the Histopathology of the Nervous

System, Rome.
(1953). Enzymologia, Amst., 16, 91. Richter, D. (1951). In Recent Progress in Psychiatry, 2nd ed. 